\section{P117 HBA1C MONITORING IN PATIENTS WITH CYSTIC FIBROSIS RELATED DIABETES (CFRD) DURING COVID 19 PANDEMIC}

M Zafran, M Yousif, D Bhaskaran, J Robinson, J Read, A Keele, K Bateman. Bristol Royal Infirmary, Bristol, UK

\subsection{6/thorax-2021-BTSabstracts.226}

Introduction Cystic fibrosis (CF) affects the pancreas, particularly the $\beta$-cells, leading to cystic fibrosis-related diabetes (CFRD) due to insulin deficiency. There is a lack of UK national guidance on how often $\mathrm{HbA1C}$ should be monitored. American Diabetes Association (ADA) recommends HbA1c should be monitored quarterly for patients with CFRD. ${ }^{1}$ For most patients with CFRD, the HbA1C treatment goal is $<7 \%$ to reduce the risk of microvascular complications. $^{1}$

We analysed clinical data for all patients with CFRD under Bristol Adult Cystic Fibrosis Centre (BACFC) over 2 years to review $\mathrm{HbA} 1 \mathrm{C}$ monitoring in patients with $\mathrm{CFRD}$.

Objectives Review if $\mathrm{HbA1C}$ is measured quarterly.

Review if HbA1C target range is achieved.

Methods 83 cases with known CFRD were included. Retrospective data was collected from CF database and ICE, recorded on excel spread sheet with patient identifiers coded and audited against ADA position statement.

Results HbA1C value of $<7 \%(<53 \mathrm{mmol} / \mathrm{mol})$ was considered as optimal control. In $2019,16 \% \quad(\mathrm{~N}=13 / 83)$ had HbA1C measured 3 times a year which dropped to $10 \%$ $(\mathrm{N}=8 / 83)$ in 2020. In 2019, 46.98\% ( $\mathrm{N}=39 / 83)$ had HbA1C checked once a year whereas in 2020 this number was $38.55 \%(\mathrm{~N}=32 / 83)$. In $2019,50 \%(\mathrm{~N}=42 / 83)$ had optimal HbA1C control; the number dropped to $42 \%(\mathrm{~N}=35 / 83)$ in 2020. Almost half of the patients i.e. 51.8\% $(\mathrm{N}=43 / 83)$ and $49.3 \%(\mathrm{~N}=41 / 83)$ were seen by diabetes team in 2019 and 2020 respectively.

CONCLUSION A small number of our patients had HbA1C checked quarterly and almost half of them had their $\mathrm{HbA} 1 \mathrm{C}$ in the target range. Adherence to ADA guidance was reduced during the COVID-19 pandemic in 2020, due to shielding and reduced face to face clinics. Almost half of the patients were seen by diabetes team and this number was not changed in 2020, although the reviews were not face to face. Action plan devised to improve service and develop a local guideline.

Developing a national guideline for CFRD monitoring should be considered, taking in to account newer technologies for diabetes monitoring.

\begin{tabular}{lll} 
Abstract P117 Table 1 & \\
\hline \begin{tabular}{lll} 
S. & Standard/Criteria & Results \\
No & & \\
\hline 1 & HbA1C measurement is recommended quarterly for patients with CFRD & $10 \%(8 /$ \\
& & $83)$ \\
2 & For most patients with CFRD, the A1C treatment goal is $<7 \%$ to reduce & $42 \%(35 /$ \\
& the risk of microvascular complications & $83)$ \\
\hline
\end{tabular}
\end{tabular}

\section{REFERENCE}

1. A Position statement of the American Diabetes Association and a Clinical practice guideline of the Cystic Fibrosis Foundation.

\section{P118 \\ THE ROLE OF PAEDIATRIC RESPIRATORY NURSE SPECIALIST IN MANAGEMENT OF NON-CF BRONCHIECTASIS}

H Smith, T Evans, P Nagakumar, M Desai, L Morris, S Stone, C Mcardle, N Parsons, K Hunjan, B Hayeer, P Kenia. Birmingham Women's Hospital, Birmingham, UK

\subsection{6/thorax-2021-BTSabstracts.227}

Background BTS 2010 bronchiectasis guideline recommends multidisciplinary care. Nurses play a vital role in bronchiectasis management. In paediatric bronchiectasis, the role of paediatric respiratory nurse specialist (RNS) is understated in literature. An informal survey asking 287 health professionals who were members of a national group showed no dedicated bronchiectasis RNS in any UK region. In 2019, RNS was allocated to take a more active role to support the paediatric bronchiectasis multi-disciplinary team (MDT), education and transition (0.6 WTE) at our centre.

Objective To appraise the current RNS role at a specialist paediatric bronchiectasis centre.

Method Prospective review of RNS role and time spent in bronchiectasis management over a 12 month period.

Results 93 CT-diagnosed bronchiectasis patients (M43:F50) with a median age of 10.05 (range:3.6-16.5) years were cared for during that time. 136 hours were spent by the RNS in supporting children and young people and families with bronchiectasis. Over 12 months, the services provided by RNSs included:

- Attendance to monthly MDT clinics (4hours per clinic) to provided support, education, and advice.

- Telephonic reviews and advice (4hours)

- Admission avoidance following remote RNS input=7 patients

- 2 patients identified suitable for home IV programme were supported (out of 9 needing IV's)

- 35 hours were spent on service development including database setup and entry.

Conclusion The RNS can support children and young people with emphasis on education, promoting adherence, remote reviews during exacerbations leading to reduced admissions, development of home treatment programmes and support transition. Nurse-led outpatient clinics can facilitate annual reviews and education. Future work involves a more formal survey to review the paediatric bronchiectasis nurses services within the UK, to develop such clinics further and a network approach with the district hospitals in West Midlands and evaluating the feedback from the families.

\section{REFERENCE}

1. British Thoracic Society guideline for non-CF bronchiectasis. Thorax, 2010;65:158

\section{P119 BREATHING PATTERN DYSFUNCTION IN PRIMARY CILIARY DYSKINESIA: MYTH OR REALITY?}

GM Housley, S Peake, M Loebinger. Royal Brompton Hospital, London, UK

\subsection{6/thorax-2021-BTSabstracts.228}

Background Breathing pattern dysfunction (BPD) is common in patients with obstructive lung disease, e.g., over $50 \%$ of patients with severe asthma show evidence of BPD. BPD is also known to affect quality of Life (QoL). Despite disparities 
Abstract P119 Table 1

\begin{tabular}{|c|c|c|c|c|c|c|c|c|c|}
\hline & $\mathrm{n}$ & Gender & Age & BPAT & RR & D-12 & SNOT-22 & EQ5D5L & EQ5D-5L Health \\
\hline & & & & $\max =14^{*}$ & & $\max =36^{*}$ & $\max =110^{*}$ & $\max =25 *$ & $\max =100 \%$ * * \\
\hline \multicolumn{10}{|l|}{ A } \\
\hline Mean & 7 & $5 F / 2 M$ & 28 & 5.42 & 15 & 15.43 & 49.14 & 2.57 & 68 \\
\hline $\pm S D$ & & & 7.1 & 0.79 & 4.12 & 10.37 & 20.97 & 2.23 & 15.75 \\
\hline \multicolumn{10}{|l|}{ B } \\
\hline Mean & 10 & $5 F / 5 M$ & 31 & 2 & 14.5 & 2.7 & 26.5 & 0.56 & 75.78 \\
\hline \pm SD & & & 21 & 0.82 & 3.57 & 2 & 12.14 & 1.13 & 18.41 \\
\hline$p=$ & & 0.2 & 0.37 & $<0.00001$ & 0.4 & 0.0008 & 0.0064 & 0.016 & 0.19 \\
\hline
\end{tabular}

data expressed as mean $\pm \mathrm{SD}$;

$\mathrm{RR}=$ respiratory rate; *higher score=worse; ${ }^{*}$ higher score=better

in definition and measurement, the Breathing Pattern Assessment Tool (BPAT) ${ }^{1}$ is increasingly being used to identify BPD. At our specialist PCD centre, we considered the relationship between possible BPD and breathlessness, sino-nasal symptoms and QoL.

Method 17 stable adult PCD patients attending virtual appointments provided lung function data using a hand-held spirometer. BPD and breathlessness were measured using the BPAT (therapist-observed) and Dyspnoea 12 (D12), a patientreported questionnaire; patients also completed the SNOT-22, which examines the impact of chronic rhinosinusitis on wellbeing, and a generic QoL measure, the EQ5D-5L. Data from patients whose BPAT score was $>4$ (group A), indicating likely BPD $^{1}$, were compared with those whose BPAT was $<4$ (Group B).

Results Age and gender distribution did not differ substantively between groups. We observed that 7/17 patients had BPD; these patients had significantly higher scores for D12, SNOT22 and EQ5D-5L (function) suggesting greater problems with breathing and rhinosinusitis, and lower QoL (table 1). However, there were no significant differences in lung function between groups $\mathrm{A}$ and $\mathrm{B}$ respectively: $\mathrm{FEV}_{1}$ (2.771 vs 2.641; $\mathrm{p}=0.42), \mathrm{FEV}_{1} \%$ pred $(76.4 \%$ vs $71.4 \% ; \mathrm{p}=0.35)$, FVC $(3.651$ vs $3.72 ; \mathrm{p}=0.46)$, FVC $\%$ pred $(84.9 \%$ vs $81.6 ; \mathrm{p}=0.36)$, $\mathrm{FEV}_{1} / \mathrm{FVC}(0.78$ vs $0.68 ; \mathrm{p}=0.12)$ and in EQ5D-5L\%health (68\% vs $76 \% ; \mathrm{p}=0.19)$.

Discussion These data suggest for the first time, that BPD is likely found in PCD, even when lung function is reasonably well maintained. The difficulties in breathing identified maybe associated with chronic rhinosinusitis and impact on patients' functional QoL. Overall perception of QoL did not differ between groups, but was only $70 \%$ of perceived maximum. Further data are needed although these results suggest the need to screen patients for BPD, target nasal symptoms aggressively and provide breathing re-education techniques.

\section{REFERENCE}

1. Todd S, Walsted E, Grillo L, et al. Novel assessment tool to detect breathing pattern disorder in patients with refractory asthma. Respirology 2017. doi:10.1111/ resp. 13173

\section{Improving care pathways in adults and children}

\section{P120 THE EFFECT OF MEDICAL FACE MASK ON ADOLESCENT CHILDREN'S OXYGEN SATURATION DURING 6-MINUTE WALK TEST}

${ }^{1}$ A Ahmad, ${ }^{2} \mathrm{M}$ Fatima, ${ }^{3} \mathrm{MZ}$ Equabal. 'Dartford Grammar School, Dartford, UK; ${ }^{2}$ Gravesend Grammar School, Gravesend, UK; ${ }^{3}$ Queen Marys Hospital, Sidcup, UK

\subsection{6/thorax-2021-BTSabstracts.229}

Aims The World Health Organization recommends that face masks are used for children aged six years and older. Children in the UK are now attending schools using face coverings and undertaking mild to moderate activity with the face covering on. We aim to study the effect of face masks on SpO2 levels of healthy adolescents with a 6-minute walk test (6MWT).

Material and Methods 11 healthy adolescent children (16-17 years) undertook the 6-minute walk test with and without a medical mask (Type IIR, triple ply) and SpO2 levels were measured with a standard commercially available pulse oximeter. A pre-walk test screening questionnaire was used to exclude children with co-morbidities. None of the screened participants needed to be excluded. Measurements included screening blood pressure, $\mathrm{SpO} 2$, pulse rate, walking distance, breathlessness and fatigue.

Results The average post 6MWT SpO2 without a mask (96.6 $\pm 1.43)$ was higher than with a mask $(94.8 \pm 4.05)$ but this was not statistically significant (t-Test $\mathrm{p}$ value 0.17 ). The average distance travelled without a mask (683.4 \pm 64.2 metres) was greater than with a mask (675.45 \pm 52 metres), again this was not a statistically significant difference (t-Test $\mathrm{p}$ value $0.75)$.

Conclusion Concerns have been raised regarding the safety of the use of face masks. ${ }^{1}$ A review regarding the impact of face masks on children has suggested the need for further studies. ${ }^{2}$ In this study we have found that the use of medical face masks does not cause a significant drop in oxygen saturations during 6MWT in healthy adolescent children. 